\title{
Ajedrez y aprendizaje del área de matemáticas en estudiantes del segundo ciclo, Estudios Generales, Universidad San Martín de Porres, 2019
}

\author{
Chess and learning in the area of mathematics in second cycle \\ students, General Studies, Universidad San Martín de Porres, \\ 2019
}

Luis Chanca Cortéz (iD Abelardo Rodolfo Campana Concha $\square$

Universidad Nacional Mayor de San Marcos. Lima, Perú

Recibido: 18/04/2021

Revisado: 18/04/2021

Aceptado: 08/05/2021

Publicado: 31/ 06 / 2021

\section{RESUMEN}

Este estudió pre experimental se propuso analizar la influencia del juego de ajedrez y aprendizaje del área matemáticas en los estudiantes universitarios, pensando en dicho fin se aplicó el pre y pos test a una muestra constituida de 40 estudiantes estudiantes segundo año de la Escuela Profesional de Administración Facultad de Ciencias Contables Económicas y Financieras de la Universidad San Martin de Porres, 2019, cuya información recolectada fue procesada con el programa SPSS, lo cual permitió aplicar prueba de diferencias de medias de $\mathrm{T}$ Student para muestras independientes. En los resultados se explica que la hipótesis general comprobó la influencia del ajedrez en el rendimiento académico del Área de Matemáticas, puesto que existe una diferencia de 1,76 puntos entre el grupo control y experimental. En las hipótesis específicas también confirmaron la influencia del ajedrez en el aprendizaje de las ecuaciones lineales, la derivada de una función, la aplicación de la derivada y el Cálculo Integral, puesto que sus diferencias de medias entre ambos grupos están en 0,49 puntos, 0,47 puntos, 1,47 puntos y 1,95 puntos, respectivamente. Por lo lo cual, se concluyó, que este actividad influye positivamente en el aprendizaje matemático y en sus dimensiones ecuaciones lineales, derivada de una función, aplicación de la derivada y cálculo integral. Palabras clave: Práctica, ajedrez, aprendizaje, matemáticas.

\section{ABSTRACT}

This pre-experimental study aimed to analyze the influence of the game of chess and learning in the area of mathematics in university students, thinking about this purpose, the pre and post test was applied to a sample of 40 second-year students from the Professional School of Administration. Faculty of Economic and Financial Accounting Sciences of the San Martin de Porres University, 2019, whose information collected was processed with the SPSS program, which allowed the application of the T Student difference test of means for independent 
samples. The results explain that the general hypothesis verified the influence of chess on the academic performance of the Mathematics Area, since there is a difference of 1.76 points between the control and experimental groups. In the specific hypotheses, they also confirmed the influence of chess on learning linear equations, the derivative of a function, the application of the derivative and the Integral Calculus, since their mean differences between both groups are 0.49 points, 0.47 points, 1.47 points and 1.95 points, respectively. Therefore, it concluded that this activity positively influences mathematical learning and its dimensions, linear equations, derivative of a function, application of the derivative and integral calculus. Keywords: Practice, chess, learning, mathematics.

\section{INTRODUCCIÓN}

Fernández y Pallarés (2009). Señalan, el juego del ajedrez es benefactor en el aprendizaje integral, ayudando el intelecto, la práctica continua mejora la mente razonando analíticamente y tomando decisiones, también como estímulo para desarrollar: atención, concentración, memoria y la intuición, se presenta como disciplina de soporte para desarrollar integralmente a los educandos.

Este nivel de importancia ha sido entendido por casi 30 países alrededor del mundo que lo promuevan en sus centros educativos a edad temprana, según Taborda (2013) resalta su interés radica en la potencie el desarrollo del pensamiento por lo cual lo incluyen el currículo. Sin embargo, en el Perú, esto aún no se ve masificado en los estudiantes, quienes crecen desconociendo este deporte desde una edad temprana, quienes llegando a una etapa adulta el interés de practicarlo. A pesar de que existen deportistas destacados de ajedrez en nuestro país, no es común su práctica.

Un artículo del diario Gestión (2016) presentó los deportes más practicados por los peruanos, sin embargo en el cuadro no se encuentra el ajedrez lo cual nos demuestra el nivel de interés de la población por este deporte.
Los resultados PISA del 2015 en el área de matemática expresaron un incremento de 18 puntos respecto a la prueba practicada en años anteriores, situación que se repitió en el PISA del 2018 el cual manifestó un incremento de 13 puntos lo cual hace un total de 400, sin embargo aún estamos en el nivel 1 de una escala de 6, ubicándonos en el puesto 64 entre 77 países.

Como vemos aún existen inconvenientes en el área de matemáticas, lo cual a una visión a futuro perjudicará en su vida universitaria, más aun teniendo alternativas didácticas como el ajedrez. Ese es nuestro interés al desarrollar esta investigación.

Existen antecedentes que anticipan esta problemática, como los de Paniagua (2017) indica que la práctica del ajedrez resulta beneficiosa para los procesos cognitivos, ya que resulta utilitaria como una herramienta fundamental para mejorar la memoria, el lenguaje, la percepción, el pensamiento y la atención de los alumnos.

Solórzano y Tariguano (2010) en su investigación entiende que los juegos lúdicos son una alternativa para el razonamiento lógico matemático, sin embargo los docentes muchas veces no lo consideran como alternativa de ense- 
ñanza, señala que la importancia de usar esta didáctica lúdica permitirá propiciar aprendizajes significativos.

Como objetivo, consiste en analizar la influencia del juego de ajedrez y aprendizaje del área de matemáticas en los estudiantes de segundo año de la Escuela Profesional de Administración Facultad de Ciencias Contables Económicas y Financieras, USMP, 2019

\section{MATERIALES Y MÉTODOS}

Es un estudio cuasi experimental ya que solo se mide y compara dos grupos (control y experimental), cuya muestra consta de 40 de estudiantes. Se les aplicó el pre y pos test para conocer si existe una influencia positiva, cuya información será procesada mediante el programa estadístico SPSS.

\section{RESULTADOS Y DISCUSIÓN}

Tabla 1. Frecuencias Pretest: Aprendizaje de la Matemática II

\begin{tabular}{llrr}
\hline \multicolumn{1}{c}{ Pretest } & Frecuencia & $\%$ \\
\hline \multirow{4}{*}{ Válidos } & Pésimo & 3 & 7,5 \\
& Malo & 14 & 35,0 \\
& Regular & 15 & 37,5 \\
& Bueno & 5 & 12,5 \\
& Muy bueno & 3 & 7,5 \\
\cline { 2 - 3 } & Total & 40 & 100,0 \\
\hline \multicolumn{3}{c}{ Fuente. Elaboración propia }
\end{tabular}

En la tabla 1 se aprecia que el 37.5\% alcanzó un nivel regular en la prueba pretest del curso de Matemática II, el 35.0\% un nivel malo, $12.5 \%$ un nivel bueno, $7.5 \%$ nivel muy bueno y un $7.5 \%$ un nivel pésimo, lo cual evidencia que los aprendizajes antes de aplicar una metodología de enseñanza no son los esperados.
Tabla 2. Frecuencias Postest: Aprendizaje de la Matemática II

\begin{tabular}{llrr}
\hline Postest & & Frecuencia & $\%$ \\
\hline \multirow{4}{*}{ Válidos } & Malo & 1 & 2,5 \\
& Regular & 15 & 37,5 \\
& Bueno & 17 & 42,5 \\
& Muy bueno & 7 & 17,5 \\
\cline { 2 - 4 } & Total & 40 & 100,0 \\
\hline \multicolumn{3}{c}{ Fuente. Elaboración propia }
\end{tabular}

En la tabla 2, el 42.5\% alcanzó un nivel bueno en la prueba pretest del curso de Matemática II, el $37.5 \%$ un nivel regular, el $17.5 \%$ un nivel muy bueno y el $2.5 \%$ un nivel malo, lo cual evidencia que luego de que los estudiantes aprendieran a jugar el ajedrez, las puntuaciones de los estudiantes mejoraron.

El proceso estadístico presentó los siguientes resultados:

HG. La práctica del juego del ajedrez incrementa el aprendizaje de las matemáticas en estudiantes del nivel superior.

Tabla 3. Diferencia medias HG

\begin{tabular}{lrrrr}
\hline $\begin{array}{l}\text { Grupo de } \\
\text { estudio }\end{array}$ & N & Media & $\begin{array}{r}\text { Desviación } \\
\text { típ. }\end{array}$ & $\begin{array}{r}\text { Error típ. } \\
\text { de la } \\
\text { media }\end{array}$ \\
\hline Control & 19 & 13,95 & 2,857 &, 655 \\
\hline Experimental & 21 & 15,71 & 1,978 &, 432 \\
\hline
\end{tabular}

Fuente. Elaboración propia

Los datos estadísticos expresados en la tabla 3 , confirman las medias logradas por el grupo de estudio, El grupo control alcanzó un promedio de 13.95, en tanto que el grupo control es de 15.71, por lo cual, la práctica del ajedrez es efectivo en el aprendizaje de la matemática porque existe una diferencia de 1.76 puntos. 
En cuanto a los planteamientos específicos, se presentan los siguientes resultados:

H1. La práctica del juego de ajedrez incrementa el aprendizaje de las ecuaciones lineales.

Tabla 4. Diferencias de medias H1

\begin{tabular}{lrrrr}
\hline $\begin{array}{l}\text { Grupo de } \\
\text { estudio }\end{array}$ & $\mathrm{N}$ & Media & $\begin{array}{r}\text { Desviación } \\
\text { típ. }\end{array}$ & $\begin{array}{r}\text { Error típ. } \\
\text { de la } \\
\text { media }\end{array}$ \\
\hline Control & 19 & 15,32 & 2,790 &, 640 \\
\hline Experimental & 21 & 15,81 & 1,887 &, 412 \\
\hline \multicolumn{5}{c}{ Fuente. Elaboración propia }
\end{tabular}

En la tabla 4, la prueba aplicada al grupo control demostraron alcanzar un promedio de 15.32, sin embargo el grupo experimental obtuvo un promedio de 15.81. Considerando que la práctica del ajedrez es efectivo en el aprendizaje de las ecuaciones lineales.

H2. La práctica del juego del ajedrez incrementa el aprendizaje de la derivada de una función en estudiantes del nivel superior.

Tabla 5. Diferencias de medias H2

\begin{tabular}{lrrrr}
\hline $\begin{array}{l}\text { Grupo de } \\
\text { estudio }\end{array}$ & $\mathrm{N}$ & Media & $\begin{array}{c}\text { Desviació } \\
\mathrm{n} \text { típ. }\end{array}$ & $\begin{array}{c}\text { Error típ. } \\
\text { de la } \\
\text { media }\end{array}$ \\
\hline Control & 19 & 15,58 & 2,950 &, 677 \\
\hline $\begin{array}{l}\text { Experiment } \\
\text { al }\end{array}$ & 21 & 16,05 & 2,109 &, 460 \\
\hline
\end{tabular}

Fuente. Elaboración propia

En la tabla 5, los resultados de la prueba aplicada al grupo control alcanzó un promedio de 15.58, sin embargo el grupo experimental obtuvo un puntaje de 16.05. Evidenciando la efectividad del ajedrez para el aprendizaje de las ecuaciones lineales.
H3. La práctica del juego de ajedrez incrementa el aprendizaje de aplicación de la derivada en estudiantes del nivel superior.

Tabla 6. Diferencia de medias H3

\begin{tabular}{lrrrr}
\hline $\begin{array}{l}\text { Grupo de } \\
\text { estudio }\end{array}$ & $\mathrm{N}$ & Media & $\begin{array}{c}\text { Desviación } \\
\text { típ. }\end{array}$ & $\begin{array}{c}\text { Error } \\
\text { típ. de } \\
\text { la } \\
\text { media }\end{array}$ \\
\hline Control & 19 & 14,53 & 3,389 &, 777 \\
\hline Experimental & 21 & 16,00 & 2,258 &, 493 \\
\hline \multicolumn{5}{l}{ Fuente. Elaboración propia }
\end{tabular}

La tabla 6, se expresa que la prueba aplicada al grupo control se observó un promedio de 14.53, en tanto el grupo experimental obtuvo un promedio de 16.00. Por ello afirmamos la efectividad de la práctica del ajedrez en el aprendizaje de aplicación de la derivada H4. La práctica del juego de ajedrez incrementa el aprendizaje de Cálculo Integral en estudiantes del nivel superior

Tabla 7. Diferencia de medias H4

\begin{tabular}{lrrrr}
\hline $\begin{array}{l}\text { Grupo de } \\
\text { estudio }\end{array}$ & N & Media & $\begin{array}{c}\text { Desviación } \\
\text { típ. }\end{array}$ & $\begin{array}{c}\text { Error } \\
\text { típ. de } \\
\text { la } \\
\text { media }\end{array}$ \\
\hline Control & 19 & 13,95 & 2,857 &, 655 \\
\hline Experimental & 21 & 15,90 & 2,322 &, 507 \\
\hline \multicolumn{4}{c}{ Fuente. Elaboración propia }
\end{tabular}

En la tabla 7, los resultados de la prueba aplicada al grupo control demostraron alcanzar un promedio de 13.95, sin embargo el grupo experimental obtuvo un promedio de 15.90. Con ello se confirma la efectividad de la práctica del ajedrez para el aprendizaje de Cálculo Integral. 
Realizando un análisis de los resultados, podemos indicar que en la hipótesis general demostró que los estudiantes que practican ajedrez tienen calificaciones más altas en el curso Matemáticas II, de los que no lo practican. Debido a que el grupo de control evidenció un promedio de 13.95 con notas en su mayoría regulares; mientras que el grupo experimental las puntuaciones registraron un promedio de 15.71 con notas buenas en su mayoría. En otras palabras, la diferencia de 1.76 entre los dos grupos evidencia la diferencia en sus calificaciones.

En la primera hipótesis específica evidenció que los estudiantes que practican ajedrez tienen calificaciones más altas en la temática de ecuaciones lineales, a diferencia de los que no lo practican. Debido a que el grupo de control manifestó calificaciones con un promedio de 15.32, con notas regulares en su mayoría; en tanto que el grupo experimental las puntuaciones registraron notas con promedio de 15.81, con notas buenas en su mayoría. En resumen, la diferencia de 0.49 entre los dos grupos evidencia relativa diferencia en sus calificaciones.

En la segunda hipótesis específica se reveló que los estudiantes que practican ajedrez tienen calificaciones más altas en la temática de derivada de una función, a diferencia de los que no lo practican. Debido a que el grupo control evidenció calificaciones con un promedio de 15.58, que en su mayoría alcanzan notas regulares; por otro lado, el grupo experimental las puntuaciones registraron un promedio 16.05, cuyas notas en su mayoría fueron buenos. En suma, la diferencia de 0.47 entre los dos grupos evidencia relativa diferencia en sus calificaciones.

En la tercera hipótesis específica se manifestó que los estudiantes que practican ajedrez tienen calificaciones más altas en la temática de aplicación de la derivada, a diferencia de los que no lo practican. Esto se explica en los resultados del grupo control con calificaciones con un promedio de 14.53 que en su mayoría era regular; en tanto que el grupo experimental las puntuaciones registraron notas con un promedio de 16.00 , quienes en su mayoría alcanzaron notas buenas. En resumen, la diferencia de 1.47 entre los dos grupos evidencia diferencias en sus calificaciones.

En la cuarta hipótesis específica se halló que los estudiantes que practican ajedrez tienen calificaciones más altas en la temática de Cálculo Integral, a diferencia de los que no saben jugarlo. Esto se entiende porque en el grupo control contó con un promedio de 13.95, con notas regulares en su mayoría; mientras que en el grupo experimental las puntuaciones registraron notas con un promedio de 15.90, con notas buenas en su mayoría. En otras palabras, la diferencia de 1.95 entre los dos grupos evidencia la diferencia en sus calificaciones.

Treviño (2015) demostró en su investigación que el ajedrez refuerza el pensamiento crítico de los estudiantes, especialmente en las habilidades de inferencia y autorregulación, aunque no se direccionó en cuanto al beneficio de algún curso en especial, la inferencia es importante para los problemas matemáticos, por ello que gurda cierta relación a nuestros resultados ya que la práctica de ajedrez incremento las notas de matemática.

Como lo hemos reiterado el ajedrez resulta importante para el aprendizaje de las matemáticas, sin embargo según Gastelu y Padilla (2017) cualquiera metodología didáctica resulta eficiente para el curso, sin embargo algo que destaca es que dependiendo de la 
metodología seleccionada no tendrá resultados positivos si es que estas no se practican con relativa constancia, no necesariamente en las aulas. El ajedrez es un actividad que se puede practicar en aula su en casa por lo cual mientras más se practique los resultados académicos serán más evidentes y beneficios, en especial en las matemáticas.

\section{CONCLUSIONES}

El primer planteamiento evidenció una diferencia entre el grupo control y experimental, cuyos alcanzaron promedios mayoritarios de regular y bueno, respectivamente; asimismo cuentan con una diferencia de medias de 1.76 . Con ello se concluye que los resultados hallados confirman que el ajedrez influye positivamente en el aprendizaje de la Matemática II.

En la propuesta específica 1 se demostró que entre el grupo control y experimental, cuyas calificaciones alcanzaron promedios de regular y bueno, respectivamente; del mismo modo cuentan con una diferencia de medias de 0.49. Por ello concluimos que la práctica del ajedrez influye en las calificaciones de los estudiantes universitarios, en este caso en el tema de las ecuaciones lineales.

Con respecto al aprendizaje de la derivada de una función, se alertó diferencias entre el grupo de control y experimental, ya que las calificaciones obtuvieron promedios de regular y bueno, respectivamente; asimismo contaron con una diferencia de medias de 0.47 . Finalmente concluimos que la práctica del ajedrez influye positivamente en las calificaciones de los estudiantes universitarios del área de Matemática, lo cual se demostró en el tema de la derivada de una función.
En referencia, al nivel de aprendizaje de la aplicación de la derivada, se encontró diferencias entre el grupo control y experimental, puesto que las calificaciones alcanzaron promedios de regular y bueno, respectivamente; de igual manera cuentan con una diferencia de medias de 1.47. En resumen, la práctica de este deporte ayuda positivamente en las notas de los estudiantes universitarios en el tema de la aplicación de la derivada.

Por otra parte, la cuarta hipótesis específica halló diferencias entre el grupo de control y experimental, cuyos promedios alcanzaron promedios mayoritarios de regular y bueno, respectivamente; del mismo modo cuentan con una diferencia de medias de 1.95. Con ello se concluye que los resultados hallados confirman que el ajedrez influye positivamente en el aprendizaje de la MatemáticaII, específicamente en los temas relacionados al Cálculo Integral.

\section{REFERENCIAS BIBLIOGRÁFICAS}

Fernández, J. y Pallarés, M. (2009). Cómo sensibilizar la escuela hacia el ajedrez [Archivo PDF]. http://dim.pangea.org/revistaDIM15/ docs/sensibilizarlaescuelahaciaelajedrez.pdf

Ministerio de Educación (2017). El Perú en PISA 2015. Informe nacional de resultados. MINEDU.

Paniagua, M. (2017). La influencia del ajedrez en los procesos cognitivos [Tesis de Maestría, Universidad Internacional de la Rioja]. https://reunir.unir.net/bitstream/handle/123456789/6288/PANIAGUA\%20BENITO $\% 2 \mathrm{C} \% 20 \mathrm{MONICA}$.pdf?sequence=1\&isAllowed $=\mathrm{y}$ 
Solórzano, J. y Tariguano, Y. (2010). Actividades lúdicas para mejorar el aprendizaje de la matemática [Tesis de Licenciatura, Universidad Estatal de Milagro]. http://repositorio. unemi.edu.ec/bitstream/123456789/1237/3/ ACTIVIDADES\%20L\%C3\%9ADICAS\%20 PARA\%20MEJORAR\%20EL\%20APRENDIZAJE\%20DE\%20LA\%20MATEM\%C3\%81TICA.pdf

Treviño, S. (2015). Ajedrez para el desarrollo del pensamiento crítico en la escuela primaria [Tesis de Maestría, Tecnológico de Monterrey]. https://www.academia.edu/21919007/ TESIS_Maestr $\% \mathrm{C} 3 \% \mathrm{ADa}$ Ajedrez_para el_desarrollo_del_pensamiento_cr\%C3\%ADtico_en_la_escuela_primaria

Gastelu, L. y Padilla, D. (2017). Influencia de los juegos didácticos en el aprendizaje del área de matemática en los alumnos de la Institución Educativa, Huaycán [Tesis de Licenciatura, Universidad Nacional de Educación]. 